\title{
BMJ Open Under-reporting of diagnosed tuberculosis to the national surveillance system in China: an inventory study in nine counties in 2015
}

\author{
Tao Li, ${ }^{1}$ Hemant Deepak Shewade, ${ }^{2,3}$ Kyaw Thu Soe, ${ }^{4}$ Jeanette J. Rainey, ${ }^{5}$ \\ Hui Zhang, ${ }^{1}$ Xin Du, ${ }^{1}$ Lixia Wang ${ }^{1}$
}

To cite: Li T, Shewade HD, Soe KT, et al. Under-reporting of diagnosed tuberculosis to the national surveillance system in China: an inventory study in nine counties in 2015. BMJ Open 2019;9:e021529. doi:10.1136/ bmjopen-2018-021529

- Prepublication history and additional material for this paper are available online. To view these files, please visit the journal online (http://dx.doi. org/10.1136/bmjopen-2018021529).

$X D$ and $L W$ contributed equally.

Received 6 January 2018 Revised 1 December 2018 Accepted 20 December 2018

D) Check for updates

(c) Author(s) (or their employer(s)) 2019. Re-use permitted under CC BY-NC. No commercial re-use. See rights and permissions. Published by BMJ.

For numbered affiliations see end of article.

Correspondence to

Dr Xin Du; duxin@chinacdc.cn

\section{ABSTRACT}

Objective The WHO estimates that almost $40 \%$ of patients diagnosed with tuberculosis (TB) are not reported. We implemented this study to assess TB under-reporting and delayed treatment registration in nine counties in China. Design A retrospective inventory study (record review). Setting Counties were selected using purposive sampling from nine provinces distributed across eastern, central and western regions of China in 2015.

Primary and secondary outcome measures Underreporting was calculated as the percentage of patients with TB not reported to TB Information Management System (TBIMS) within 6 months of diagnosis. Delayed registration was estimated as the percentage of reported cases initiating treatment 7 or more days after diagnosis. Multivariable logistic regression and an alpha level of 0.05 were used to examine factors associated with these outcomes.

Results of the 5606 patients with TB identified from project health facilities and social insurance systems, 1082 (19.3\%) were not reported to TBIMS. Of the 4524 patients successfully reported, 1416 (31.3\%) were not registered for treatment within 7 days of diagnosis. Children, TB pleurisy, patients diagnosed in the eastern and central regions and patients with a TB diagnosis recorded in either health facilities or social insurance system - but not both-were statistically more likely to be unreported. Delayed treatment registration was more likely for previously treated patients with TB, patients with negative or unknown sputum results and for patients diagnosed in the eastern region.

Conclusion Almost one in every five patients diagnosed with TB in this study was unknown to local or national TB control programmes. We recommend strengthening TB data management practices, particularly in the eastern and central regions, and developing specific guidelines for reporting paediatric TB and TB pleurisy. Patient education and follow-up by diagnosing facilities could improve timely treatment registration. Additional studies are needed to assess under-reporting elsewhere in China.

\section{INTRODUCTION}

The WHO estimates that almost $40 \%$ of all patients with tuberculosis (TB) are not reported to local or national TB surveillance
Strengths and limitations of this study

- We conducted a retrospective inventory study following the guidelines outlined by WHO.

- Including social insurance system data provided additional information on tuberculosis (TB) case reporting and treatment registration.

- Our findings are unlikely to be representative for other locations in China.

- Inaccurate recording by health/treatment facilities could have negatively affected our linkage process.

- We only focused on healthcare facilities with TB diagnostic capability and reporting at least one TB cases during the year-long study period.

programmes each year. ${ }^{1}$ TB under-reporting can occur if patients are not diagnosed or are not reported to national TB surveillance systems following diagnosis. Under-reporting can result in inaccurate TB burden estimates. Additionally, delays in treatment initiation can prolong morbidity and increase the chances of subsequent transmission. ${ }^{23}$ Both under-reporting and a delay in initiating treatment can negatively affect the evidence base needed for prioritising resources for TB elimination programmes.

Although the number of new TB cases in China has declined substantially since $2009,{ }^{4}$ the country continues to experience a substantial burden of TB with approximately 895000 incident cases a year, or $10 \%$ of all new cases globally. ${ }^{1}$ In China, all suspected TB cases are clinically diagnosed or bacteriologically confirmed and managed in government-appointed local Centers for Disease Control and Prevention (CDC), TB dispensaries or TB-designated hospitals. Patients seeking care at other locations are referred to TB dispensaries and hospitals. Since 2005, staff in TB health facilities have been required to report diagnosed patients (clinically or 


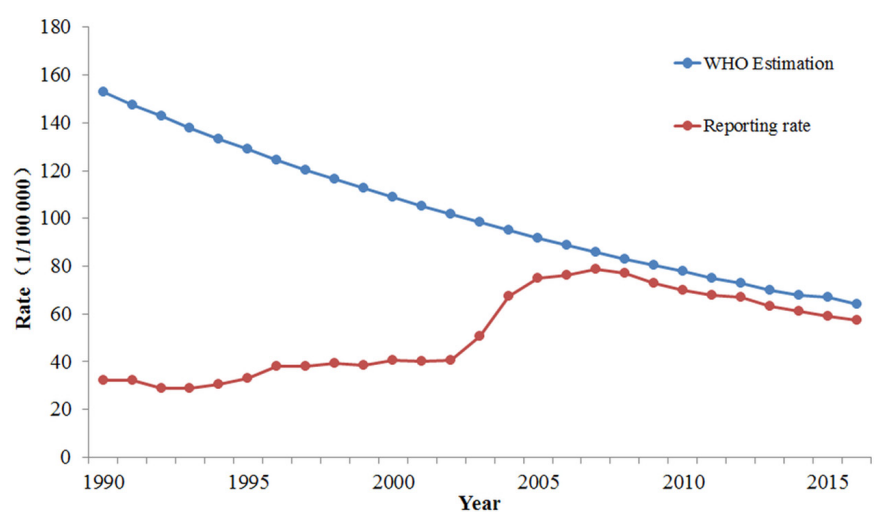

Figure 1 Estimated incidence and reporting rate to TB Information Management System (TBIMS) of TB of China, 1990-2015 (Source: WHO TB Reports, China's TBIMS system).

laboratory confirmed) directly into a web-based TB Information Management System (TBIMS), which serves as the national TB surveillance system. ${ }^{5}$ At least one TB-designated hospital or dispensary in all about 3000 counties in China has the ability to connect to TBIMS. Despite this coverage, WHO estimates that up to 120000 patients may not have been reported in $2016 .{ }^{1}$ Although the gap between estimated and reported patients has narrowed (figure 1), ${ }^{6}$ more information is needed to validate under-reporting and describe factors associated with these estimates.

The WHO recommends that all high TB-burden countries conduct 'Inventory Studies' to assess under-reporting of patients diagnosed with TB to national surveillance systems. ${ }^{7}$ Several countries, including Kenya, ${ }^{8}$ Pakistan, ${ }^{9}$ Egypt $^{10}$ and Iraq, ${ }^{11}$ have conducted inventory studies to identify and address gaps in TB reporting. Because China is a high TB-burden country, the National Center for TB Prevention and Control (NCTB) implemented a study to estimate the magnitude of TB under-reporting and treatment registration timeliness. In this paper, we report the methods and results from this assessment conducted in nine high-risk provinces in 2015. We anticipate that our findings can be used to help improve both the accuracy of TB case reporting and timely registration for treatment programmes.

\section{METHODS \\ Design}

We conducted a retrospective inventory study following guidelines outlined by the WHO. ${ }^{7}$ These guidelines describe an approach for identifying and comparing patients diagnosed with $\mathrm{TB}$ with official national $\mathrm{TB}$ case reports. We estimated the percentage of diagnosed patients using hospital records and social insurance system data that were reported to TBIMS. We also estimated the percentage of diagnosed patients who were registered for treatment in a timely manner.

\section{Setting}

China is divided into 34 provinces (including three special administrative regions) which are further divided into 2851 counties, and the estimated population was 1.38 billion in $2016 .{ }^{12}$ According to 2016 surveillance data, the annual estimated incidence rate of TB was approximately 64 patients per 100000 population. ${ }^{1}$ High-incidence provinces were primarily located in western China where the level of socioeconomic development typically lags behind the national average.

NCTB is responsible for coordinating the national TB programme, including managing TBIMS. Provincial and county-level institutions oversee the design and implementation of $\mathrm{TB}$ prevention, control and treatment services. Residents who have a presumptive or confirmed TB diagnosis will first be reported to the national infectious disease surveillance system, and then referred to a Basic Management Unit (BMU) located within county-level health facilities (county-level CDC, TB dispensaries and designated hospitals). As outlined in the national guidelines, patient's TB examinations include a sputum smear test and chest radiograph or chest X-ray (CXR) ${ }^{13}$ BMUs are required to report and register all patients diagnosed and treated with TB (bacteriologically and clinically confirmed) along with patient outcomes (ie, recovered, died, lost to follow-up) to the NCTB using TBIMS. ${ }^{13}$

In China, healthcare costs are covered by patient self-pay, private health insurance from the patient's employer or China's social insurance system. Almost 95\% of the population uses the social insurance system; this system registers clinician-based TB diagnoses and can be used to identify patients. Migrant workers-those who travel from one county to another for employment opportunities and are there for less than 6 months-are only able to access the social insurance system in their official place of residence.

\section{Site selection}

For this initial study, we used purposive sampling to select nine provinces from eastern, central and western China. These were selected to represent high, moderate and low levels of socioeconomic development, rather than specific geographic regions in the country. The nine provinces included Jiangsu, Zhejiang and Shanghai from the eastern region (high socioeconomic-level provinces); Henan, Heilongjiang and Hubei from the central region (middle socioeconomic-level provinces); and Guangxi, Sichuan and Yunnan from the western region (low socioeconomic-level provinces).

We selected one county from each of these provinces, for a total of nine counties, to participate in the project. Each selected county had a high TB burden and demonstrated the capacity to conduct the study, including availability of technical staff, proven commitment and strong TB programme leadership (figure 2). In each county, we included healthcare facilities that were able to perform CXR and that had diagnosed at least one patient with TB 


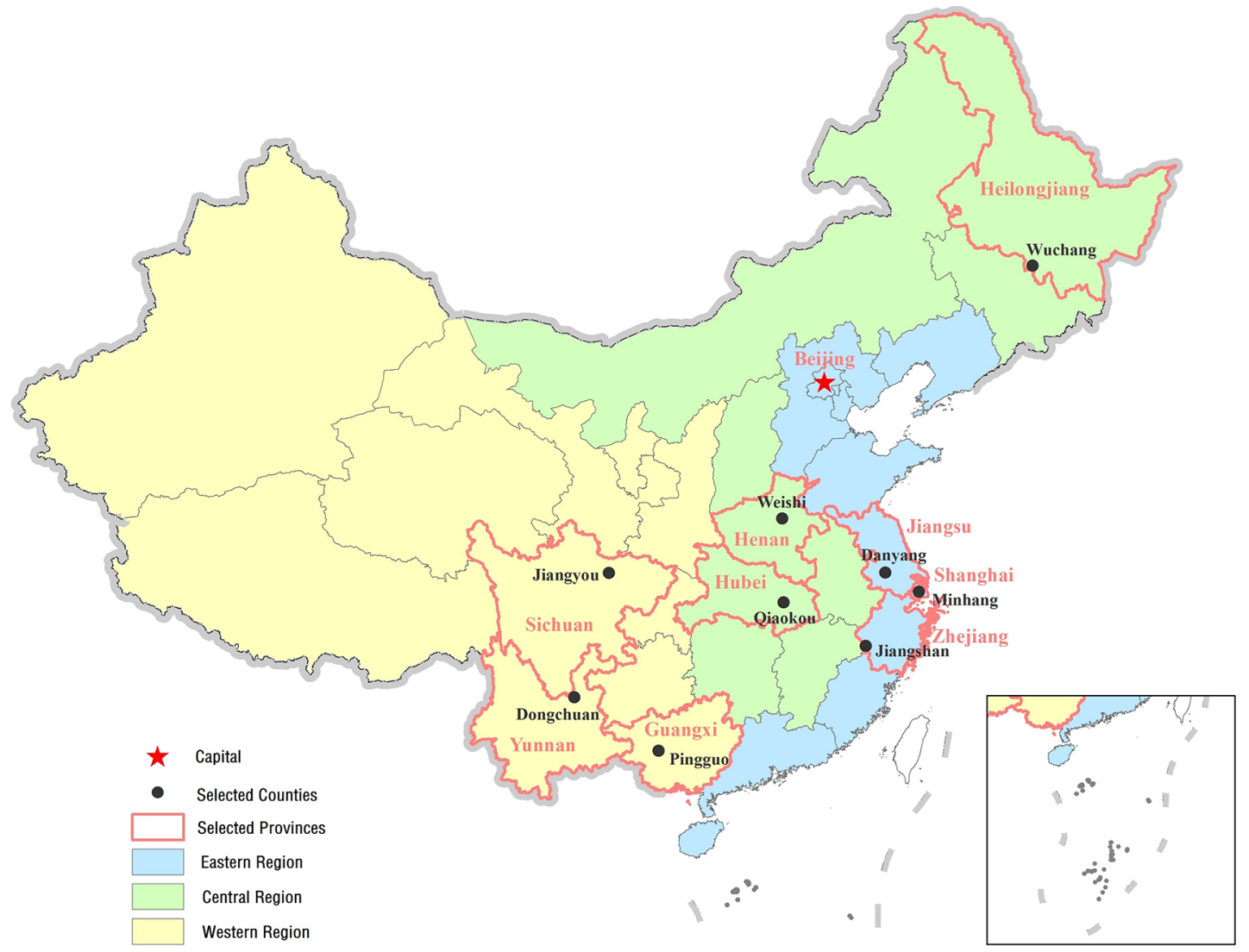

(C) 2018 CHINA CDC - Infectious Disease Reporting System

Figure 2 Location of counties and provinces selected through purposive sampling for the tuberculosis (TB) under-reporting study, by region, China, 2015.

during the project period. Facilities not meeting these criteria were excluded. This approach could provide a good initial estimate of the level of under-reporting in each region, while limiting the complexities associated with identifying a generalisable sample through cluster sampling or other methodologies.

\section{Inclusion criteria for patients with TB}

We included patients with TB if the patient's medical record indicated a clinician-based TB diagnosis, based on national TB diagnostic guidelines, ${ }^{1314}$ or if the social insurance system had documentation of the patient receiving a TB diagnosis. We obtained information on patients diagnosed with pulmonary TB (PTB) disease or TB pleurisy between 1 January 2015 and 31 December 2015.

\section{Data collection}

We reviewed medical records at the participating health facilities and county-level social insurance system databases to identify patients diagnosed with TB. ${ }^{13}{ }^{14}$ If an electronic medical record system was available, we exported all records with a TB diagnosis to an Excel spreadsheet. If only paper records were available, we performed a manual review of medical records from outpatient departments, inpatient wards and laboratory reports for a TB diagnosis. We also searched the social insurance system electronic databases for patients with health facility visit dates in 2015 and a TB (PTB) or TB pleurisy diagnosis. Reports meeting these criteria were exported and added to the medical record Excel spreadsheet. The following information was captured and included in the medical record spreadsheet: name, age, sex, national identification (ID) number, date of diagnosis, address and type of TB (pulmonary/pleurisy). Duplicate records were removed. The medical record spreadsheet was imported into an Excel data set for the linkage analysis.

From TBIMS, we downloaded patients' personal information (name, age, sex, national ID number and address) into a separate Excel data set. We also captured the date of diagnosis, date of registration, sputum status for patients diagnosed with PTB and TB category (new or previously treated). Because hospital staff members are not required to report extrapulmonary $\mathrm{TB}$ to TBIMS, and treatment regimens for multidrug-resistant TB (MDR-TB) may involve different payment schemes, we excluded patients with extrapulmonary TB and MDR-TB from both data sets.

\section{Record linkage}

We used multiple approaches to link TB case data from the medical record data set with the TBIMS data set. We initially used the national ID number, name, age and 
gender to match patients. If we identified a match using the national ID, we verified this match using name, gender and age; if at least one of the three variables matched, then the link was maintained. If a national ID number was missing from one or both data sets, we attempted to match the name of each patient in the TBIMS file to the name in the medical record data set. Records with name matches were further assessed by age ( \pm 2 years), gender and address (if the addresses in the two databases were not mutually exclusive; eg, if the address in one database only had the name of prefecture name that was linked with a detailed address within the prefecture in the other database). Because some patients may seek medical care at a location away from their place of residence, we also searched provincial and national-level TBIMS files for matches to the residential address listed in the medical record data set.

Based on the above approaches, we defined a link when a TB case from the medical record and the TBIMS data set met the following conditions: (1) the national ID number matched and name, age or gender were the same; or (2) the name, age, gender and location (county/district, township, street) matched. The remaining patients with TB in the medical record data set who did not meet these conditions were defined as under-reported. Thirty-two project members were involved in the record linkage process. The final linked data set is included in the online supplementary S1 annex.

\section{Data analysis}

We described the characteristics of patients with TB identified from the participating healthcare facilities and social insurance systems. We calculated under-reporting as the number and percentage of patients with TB not reported to TBIMS within 6 months of diagnosis. Delayed registration was estimated as the number and percentage of reported cases registering for treatment 7 or more days after diagnosis. We used logistic regression analysis to examine the role of the patient and health system characteristics in TB under-reporting and delayed treatment initiation. Variables with a $\mathrm{p}<0.20$ in our crude analysis were included in the multivariable model. For these analyses, we grouped each of the nine counties into one of the three socioeconomic development regions (ie, eastern, central or western region). Age was defined as the patient's age (in years) at the time of TB diagnosis. Patients aged $\geq 65$ years and diagnosed in the western region served as the reference group. Crude OR and adjusted OR (aOR) and corresponding 95\% CIs were generated. An alpha level of 0.05 was used to assess statistical significance. We performed all analyses using STATA (V.12.1, StataCorp, College Station, TX, USA).

\section{Patient and public involvement}

Project staff did not interact directly with patients with TB during this retrospective record review. We worked with administrators and staff at the selected hospitals as well as the social insurance system offices in the nine provinces.
Findings from this study will help TB-designated hospitals as well as provincial and national TB programmes to improve patient care and treatment.

\section{RESULTS}

We identified a total of 259 health facilities in the nine project counties. Among these, 149 (49.7\%) met our health facility inclusion criteria (ie, able to perform CXR and diagnosed at least one patient with $\mathrm{TB}$ during the project period). We reviewed a total of 61083 medical records: 54557 medical records from health facilities and 6526 records from social insurance systems. At the time of the study, 37 (24.8\%) facilities were using paper medical records. A total of 5606 records with a PTB or TB pleurisy diagnosis were identified from these reviews and included in our analysis (table 1, figure 3 ).

Of the identified patients with TB, $4178(74.5 \%)$ were 15-64 years of age and $3918(69.9 \%)$ were male. We identified eligible patients diagnosed with TB primarily from healthcare facility medical records $(\mathrm{n}=3358,59.9 \%$ ) and a smaller percentage from social insurance systems $(\mathrm{n}=896,16.0 \%)$; almost one-quarter $(\mathrm{n}=1352,24.1 \%)$ were identified from both sources. Of all eligible TB cases, $2702(48.2 \%)$ were identified from three counties (Wuchang, Heilongjiang Province, 1108 (19.8\%), Minhang, Shanghai, 839 (15.0\%) and Qiaokou, Hubei Province, $755(13.5 \%))$.

Of the 5606 TB cases identified from the nine counties, $1082(19.3 \%)$ cases were not reported to TBIMS within 6 months of diagnosis. Under-reporting ranged from $398(23.2 \%)$ TB cases in the eastern region and 556 $(21.6 \%)$ in the central region, to $128(9.7 \%)$ TB cases in the western region. Among the remaining identified TB cases, $5402(96.4 \%)$ were matched by national ID, patient name, age, gender and address in county-level TBIMS data set and $204(3.6 \%)$ were matched to the provincial or national TBIMS data set.

Of the 4524 patients reported to TBIMS, 1416 (31.3\%) patients with $\mathrm{TB}$ were not registered for treatment within 7 days, despite an overall median time to registration of 3 days $(\mathrm{IQR}=11$; $\mathrm{Q} 1=1, \mathrm{Q} 3=12$ ) (online supplementary figure S2). Over $30 \%$ of patients diagnosed with TB with negative or unknown sputum results were not registered for treatment.

In our crude analysis of factors associated with under-reporting, age, recording source (health facility, social insurance system or both), type of TB (PTB or TB pleurisy) and region were statistically significant (table 3 ). In our multivariable analysis, these variables remained statistically significant; under-reporting was statistically more likely for patients with paediatric TB who were $<15$ years of age (aOR 7.3, 95\% CI 3.6 to 14.9), patients whose diagnosis was recorded only in the social insurance system (aOR 22.4, 95\% CI 16.7 to 30.1), patients diagnosed with TB pleurisy (aOR 4.5, 95\% CI 3.4 to 6.0 ) and patients diagnosed in the eastern region (aOR 6.0, 95\% CI 4.7 to 7.7) or central region (aOR 2.3, 95\% CI 1.8 to 2.9 ). 
Table 1 Characteristics of patients diagnosed with pulmonary TB or TB pleurisy whose data were captured from health facility medical records and social insurance systems from nine counties in China, 2015

\begin{tabular}{|c|c|}
\hline \multirow[b]{2}{*}{ Characteristic } & \multirow{2}{*}{$\begin{array}{l}\text { Patients with TB } \\
\text { diagnosis } \\
\mathrm{n}(\%)\end{array}$} \\
\hline & \\
\hline Total & $5606(100)$ \\
\hline \multicolumn{2}{|l|}{ Age (years) } \\
\hline$<15$ & $36(0.6)$ \\
\hline $15-64$ & $4178(74.5)$ \\
\hline$\geq 65$ & $1392(24.8)$ \\
\hline \multicolumn{2}{|l|}{ Gender } \\
\hline Female & $1688(30.1)$ \\
\hline Male & 3918 (69.9) \\
\hline \multicolumn{2}{|l|}{ Recording system* } \\
\hline Health facilities & 3358 (59.9) \\
\hline Social insurance systems & $896(16.0)$ \\
\hline Both & $1352(24.1)$ \\
\hline \multicolumn{2}{|l|}{ Type of TB } \\
\hline Pleurisy & $271(4.8)$ \\
\hline PTB & 5335 (95.2) \\
\hline \multicolumn{2}{|l|}{ Region } \\
\hline Eastern & $1719(30.7)$ \\
\hline Danyang, Jiangsu & $459(8.2)$ \\
\hline Minhang, Shanghai & $839(15.0)$ \\
\hline Jiangshan, Zhejiang & $421(7.5)$ \\
\hline Central & $2573(45.9)$ \\
\hline Weishi, Henan & $710(12.7)$ \\
\hline Wuchang, Heilongjiang & $1108(19.8)$ \\
\hline Qiaokou, Hubei & 755 (13.5) \\
\hline Western & $1314(23.4)$ \\
\hline Pingguo, Guangxi & $416(7.4)$ \\
\hline Jiangyou, Sichuan & $685(12.2)$ \\
\hline Dongchuan, Yunnan & $213(3.8)$ \\
\hline
\end{tabular}

*Source of information on TB diagnosis.

PTB, pulmonary TB; TB, tuberculosis.

Among patients reported to TBIMS within 6 months, delayed registration was statistically associated with a recording source, reporting category (new or previously treated) and the region in our crude analysis (table 4). In our multivariable analysis, delayed registration was more likely for patients previously treated with $\mathrm{TB}$ (aOR $1.7,95 \%$ CI 1.4 to 2.1 ), and marginally more likely for patients with negative or unknown sputum results (aOR 1.2, 95\% CI 1.1 to 1.5) and for patients diagnosed in the eastern region (aOR 1.2, 95\% CI 1.1 to 1.5). Delayed registration was significantly less likely for patients with $\mathrm{TB}$ recorded only in the social insurance system (aOR 0.7 , $95 \%$ CI 0.5 to 0.9$)$.
Table 2 Number of days to registration in the National TB Information Management System after TB diagnosis among patients registered in nine counties in China in 2015

\begin{tabular}{|c|c|c|}
\hline Variables & n (\%) & $\begin{array}{l}\text { Median (IQR: } \\
\text { Q1, Q3) }\end{array}$ \\
\hline Total & 4524 (100.0) & $3(11: 1,12)$ \\
\hline \multicolumn{3}{|l|}{ Age (years) } \\
\hline$<15$ & $15(0.3)$ & $3(33: 0,33)$ \\
\hline $15-64$ & $3330(73.6)$ & $3(11: 1,12)$ \\
\hline$\geq 65$ & $1179(26.1)$ & $3(12: 1,13)$ \\
\hline \multicolumn{3}{|l|}{ Sex } \\
\hline Female & 1353 (29.9) & $3(12: 1,13)$ \\
\hline Male & $3171(70.1)$ & $3(10: 1,11)$ \\
\hline \multicolumn{3}{|l|}{ Recording system } \\
\hline Health facilities & 2757 (60.9) & $4(13: 1,14)$ \\
\hline Social insurance systems & $508(11.2)$ & $1(4: 0,4)$ \\
\hline Both & $1259(27.8)$ & $3(7: 1,8)$ \\
\hline \multicolumn{3}{|l|}{ Type of TB* } \\
\hline $\begin{array}{l}\text { Sputum-negative/unknown } \\
\text { PTB }\end{array}$ & $2987(66.0)$ & $3(12: 1,13)$ \\
\hline Pleurisy & $150(3.3)$ & $2(9: 0,9)$ \\
\hline Sputum-positive PTB & $1387(30.7)$ & $3(1,10)$ \\
\hline \multicolumn{3}{|l|}{ TB treatment category } \\
\hline Previously treated & $406(9.0)$ & $5(18: 1,19)$ \\
\hline New & 4118 (91.0) & $3(10: 1,11)$ \\
\hline \multicolumn{3}{|l|}{ Region } \\
\hline Eastern & $1321(29.2)$ & $3(10: 1,11)$ \\
\hline Danyang, Jiangsu & $419(9.3)$ & $2(6: 0,6)$ \\
\hline Minhang, Shanghai & $522(11.5)$ & $8(21: 2,23)$ \\
\hline Jiangshan, Zhejiang & $380(8.4)$ & $3(3: 1,4)$ \\
\hline Central & 2017 (44.6) & $3(13: 0,13)$ \\
\hline Weishi, Henan & $667(14.7)$ & $9(28: 2,30)$ \\
\hline Wuchang, Heilongjiang & $701(15.5)$ & $0(1: 0,1)$ \\
\hline Qiaokou, Hubei & $649(14.3)$ & $6(11: 4,15)$ \\
\hline Western & $1186(26.2)$ & $3(8: 1,9)$ \\
\hline Pingguo, Guangxi & $358(7.9)$ & $4(15: 1,16)$ \\
\hline Jiangyou, Sichuan & $664(14.7)$ & $3(5: 1,6)$ \\
\hline Dongchuan, Yunnan & $164(3.6)$ & $1(6: 0,6)$ \\
\hline
\end{tabular}

*Sputum smear status for PTB was available in TB Information Management System (TBIMS) for all patients registered for treatment.

†TB treatment category was available in TBIMS for all patients registered for treatment.

PTB, pulmonary TB; Q1, first quartile; Q3, third quartile; TB, tuberculosis.

\section{DISCUSSION}

This study, based on the WHO's guidelines for conducting TB inventory studies, determined that almost one in five patients diagnosed with TB in the participating counties was not reported to TBIMS. Additionally, among patients 


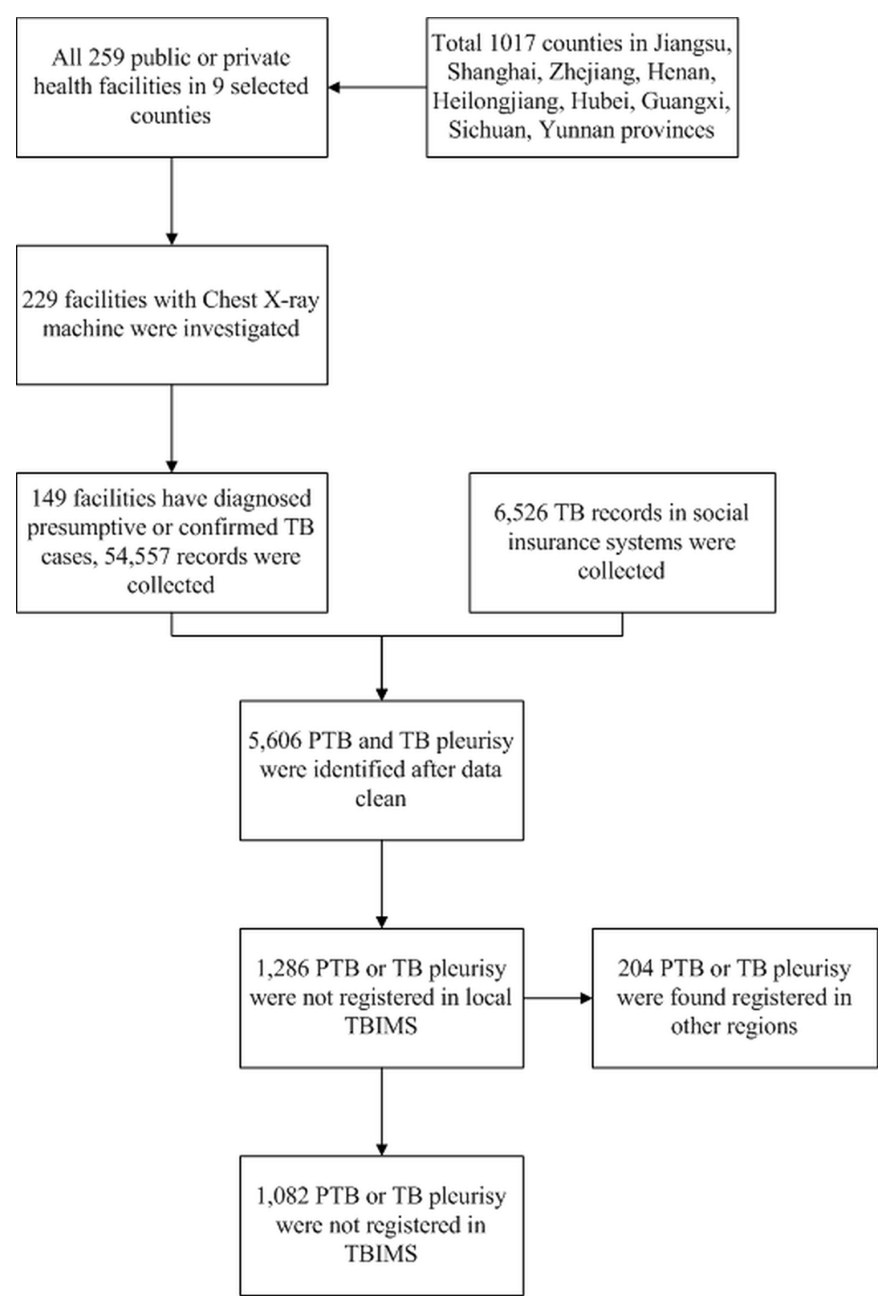

Figure 3 Flow diagram depicting each step of data collection, China, 2015. PTB, pulmonary TB; TB,

tuberculosis; TBIMS, TB Information Management System.

successfully reported, one in three was not registered for treatment within 7 days following diagnosis. Therefore, the overall TB burden in these counties is likely to be underestimated, and many patients may be at risk of prolonged morbidity and contribute to unnecessary TB transmission.

Our findings are similar to inventory studies conducted in other countries. ${ }^{8-1115-18}$ Globally, the percentage of TB cases that are unreported has ranged from $15 \%$ in European countries, ${ }^{15}{ }^{17} 20 \%$ in Africa ${ }^{8}$ and $30 \%$ in the WHO Eastern Mediterranean Region ${ }^{10} 11{ }^{16}$ to $50 \%$ in some countries with a large private sector in Asia. ${ }^{9} 18$ These estimates may reflect differences in the national TB burden, prevalence of HIV/AIDS and role of the public and private sectors in providing healthcare services. ${ }^{19}$ In our study, under-reporting was associated with region (ie, socioeconomic development level), type of TB and age (paediatric TB).

High under-reporting in eastern counties, such as Minhang in Shanghai Province, and to a lesser extent in central counties, such as Wuchang in Heilongjiang Province, may be associated with the overall size (ie, number of inpatient beds) of the designated TB hospital. Several large national and regional reference hospitals that also serve as TB hospitals are in or covered by these counties, each with more than thousand inpatient beds. The high workload and limited resources for extra staff in these hospitals could negatively affect reporting practices. This is supported by findings from studies in Kenya, where larger facilities and high patient case loads were described as possible reasons for unreported TB cases. ${ }^{8}$ Additionally, staff at certain hospitals in the eastern and central regions may prefer to receive culture confirmation before entering data of patients with TB into TBIMS to reduce time for case reporting. Qualitative projects, such as interviews of TB-designated hospital staff, are needed to better understand the causes of under-reporting at these large facilities.

In our project, under-reporting was also associated with the TB record source (ie, from the health facility or social insurance system). Identifying records of patients with TB in only one of the two recording sources suggests that the patient may have been a migrant worker. If a patient's TB diagnosis was only recorded at the health facility, but not in the social insurance system, the patient was probably not a local resident in the hospital's catchment area, and therefore could not be reimbursed. Similarly, if a patient was only recorded in the social insurance system but not at the health facility, the patient may have never been seen or diagnosed at the project facility, but, rather, was diagnosed in another place and returned home for reimbursement from the social insurance system. ${ }^{20}$ Several pilot projects have been implemented to facilitate crosscountry reimbursement under the concept of Universal Health Coverage. Follow-up studies should be conducted to assess the impact of these projects on $\mathrm{TB}$ reporting and treatment registration.

Although the total number of patients with paediatric TB and TB pleurisy identified in our project was small, under-reporting was high for both groups. Paediatric patients may not present with typical TB symptoms ${ }^{21-23}$ and current reporting regulations for TB pleurisy vary by province. ${ }^{24}$ Additionally, most paediatric patients are treated at paediatric hospitals or large general hospitals, which are not directly connected to TBIMS. Linking these hospitals to TBIMS could help better understand and monitor paediatric TB in China.

In China, all patients reported in TBIMS are required to initiate treatment as soon as possible to prevent further transmission. This is consistent with the WHO's TB treatment recommendation. ${ }^{25}$ The overall median time from TB diagnosis to treatment initiation in our study was 3 days, which is shorter than that reported by studies conducted in Tanzania and Ethiopia, ${ }^{26}{ }^{27}$ but similar to studies in India, ${ }^{28}$ and longer than a study conducted in Shandong Province, China. ${ }^{29}$ Delays in treatment registration and initiation in these studies were linked to lower symptom severity, longer travel distance to a facility, lower income, being a member of a migrant population and a lower knowledge level about TB.

Patients with sputum-negative or unknown test results and with a history of treatment were also associated with a 
Table 3 Factors associated with under-reporting to the National TB Information Management System within 6 months of TB diagnosis from nine counties in China in 2015

\begin{tabular}{|c|c|c|c|c|}
\hline Variable & Total & Under-reporting n (\%) & Crude OR(95\% Cl) & Adjusted OR $(95 \% \mathrm{Cl})$ \\
\hline Total & 5606 & $1082(19.3)$ & - & \\
\hline \multicolumn{5}{|l|}{ Age (years) } \\
\hline$<15$ & 36 & $21(58.3)$ & $7.7(3.9 \text { to } 15.3)^{\star}$ & $7.3(3.6 \text { to } 14.9)^{\star}$ \\
\hline $15-64$ & 4178 & $848(20.3)$ & $1.4(1.2 \text { to } 1.7)^{\star}$ & 1.2 (1.0 to 1.4$)$ \\
\hline$\geq 65$ & 1392 & $213(15.3)$ & Ref & Ref \\
\hline \multicolumn{5}{|l|}{ Sex } \\
\hline Female & 1688 & $335(19.8)$ & 1.1 (0.9 to 1.2$)$ & $1.0(0.9$ to 1.20$)$ \\
\hline Male & 3918 & 747 (19.1) & Ref & Ref \\
\hline \multicolumn{5}{|l|}{ Recording system $†$} \\
\hline Health facilities & 3358 & 601 (17.9) & $3.0(2.3 \text { to } 3.7)^{\star}$ & $4.3(3.4 \text { to } 5.5)^{\star}$ \\
\hline Social insurance systems & 896 & 388 (43.3) & $10.3(8.1 \text { to } 13.3)^{*}$ & $22.4(16.7 \text { to } 30.1)^{\star}$ \\
\hline Both & 1352 & $93(6.9)$ & Ref & Ref \\
\hline \multicolumn{5}{|l|}{ Type of TB } \\
\hline Pleurisy & 271 & $121(44.6)$ & $3.7(2.9 \text { to } 4.7)^{\star}$ & $4.5(3.4 \text { to } 6.0)^{\star}$ \\
\hline PTB & 5335 & $961(18.0)$ & Ref & Ref \\
\hline \multicolumn{5}{|l|}{ Region‡ } \\
\hline Eastern & 1719 & $398(23.2)$ & $2.8(2.3 \text { to } 3.5)^{\star}$ & $6.0(4.7 \text { to } 7.7)^{\star}$ \\
\hline Central & 2573 & $556(21.6)$ & $2.6(2.1 \text { to } 3.1)^{\star}$ & $2.3(1.8 \text { to } 2.9)^{\star}$ \\
\hline Western & 1314 & $128(9.7)$ & Ref & Ref \\
\hline
\end{tabular}

*Statistically significant.

†Source of information on TB diagnosis.

$\ddagger$ Based on socioeconomic development level: high level in eastern region, medium level in central region and low level in western region.

PTB, pulmonary TB; TB, tuberculosis.

delay in treatment initiation in our multivariable analysis. A negative sputum or unknown test result could suggest lower TB symptom severity while previously treated patients with TB may reflect an unfavourable view of the 6-8 month long treatment and a reluctance to reinitiate the treatment. The lack of perceived treatment effectiveness and concerns about side effects could also cause a delay in reinitiating treatment. In Shaanxi Province, for example, newly diagnosed patients sought care and treatment significantly sooner following symptom onset compared with previously treated patients with TB. ${ }^{30}$ Because of increasing occurrence of MDR-TB, ${ }^{31}$ ensuring that patients receive appropriate treatment regimens along with new strategies for successful implementation of direct observed therapy is required. ${ }^{30}$

Delayed treatment registration was highest in Minhang District in the eastern region (data not shown). Hospitals in Minhang often serve migrant workers who are likely to be diagnosed with TB near their employment site (ie, in Minhang District), but who seek treatment in their home province where the cost of TB drugs is covered by the National TB Control Program or the social insurance system. ${ }^{32-34}$ In our linkage analysis, 204 patients were diagnosed and linked to TBIMS after reviewing of reports from other provinces, including 59 patients with TB diagnosed in Minhang District. We are unable to explain the lower likelihood of delayed treatment registration for records of patients with TB identified solely from the social insurance system. Additional analyses are needed to further evaluate this finding.

The overall goal of this project and other similar inventory studies was to assess the capacity of the existing surveillance system to accurately report patients diagnosed with $\mathrm{TB}$ and to assess treatment registration and patient outcomes. ${ }^{7}$ According to the national guidelines in China, BMU staff are responsible for case reporting and treatment registration using TBIMS. We noted a large number of patients with TB who were identified from social insurance systems who were not documented in the medical records as required. Future capture-recapture (CR) studies using patient data from TBIMS, medical records and the social insurance systems could be conducted to help understand this discrepancy. ${ }^{7} \mathrm{CR}$ studies have been implemented in Egypt and Iraq using three sources of TB information to estimate under-reporting and to indirectly calculate incidence..$^{10}{ }^{11}$ Additionally, automated linkages between the social insurance system and TBIMS could be developed to provide additional methods to improve reporting, assuming that this could include procedures, using machine language and probabilistic models - to identify duplicates and to accurately match patients. ${ }^{35} 36$ 
Table 4 Factors associated with delayed ( $\geq 7$ days) treatment registration among patients diagnosed with TB reported to the National TB Information Management System from nine counties in China in 2015

\begin{tabular}{|c|c|c|c|c|}
\hline Characteristics & Total & $\begin{array}{l}\text { Delayed registration } \\
\mathrm{n}(\%)\end{array}$ & $\begin{array}{l}\text { Crude OR } \\
(95 \% \mathrm{Cl})\end{array}$ & $\begin{array}{l}\text { Adjusted OR } \\
(95 \% \mathrm{Cl})\end{array}$ \\
\hline Total & 4524 & $1416(31.3)$ & & \\
\hline \multicolumn{5}{|l|}{ Age (years) } \\
\hline$<15$ & 15 & $6(40)$ & $1.4(0.5$ to 4.0$)$ & $1.2(0.4$ to 3.5$)$ \\
\hline $15-64$ & 3330 & $1032(31.0)$ & 1.0 (0.8 to 1.1$)$ & 0.9 (0.8 to 1.1$)$ \\
\hline$\geq 65$ & 1179 & $378(32.1)$ & Ref & Ref \\
\hline \multicolumn{5}{|l|}{ Sex } \\
\hline Female & 1353 & $429(31.7)$ & 1.0 (0.9 to 1.2$)$ & 1.0 (0.9 to 1.2$)$ \\
\hline Male & 3171 & $987(31.1)$ & Ref & Ref \\
\hline \multicolumn{5}{|l|}{ Recording system $†$} \\
\hline Heath facilities & 2757 & $997(36.2)$ & $1.6(1.4$ to 1.9$)$ & $1.7(1.4 \text { to } 2.0)^{*}$ \\
\hline Social insurance systems & 508 & $97(19.1)$ & 0.7 (0.5 to 0.9$)$ & $0.7(0.5 \text { to } 0.9)^{*}$ \\
\hline Both & 1259 & $322(25.6)$ & Ref & Ref \\
\hline \multicolumn{5}{|l|}{ Type of TB } \\
\hline Sputum-negative/unknown PTB & 2987 & $966(32.3)$ & $1.2(1.0$ to 1.3$)$ & $1.2(1.1 \text { to } 1.5)^{\star}$ \\
\hline Pleurisy & 150 & $45(30.0)$ & 1.0 (0.7 to 1.5$)$ & $1.2(0.8$ to 1.7$)$ \\
\hline Sputum-positive PTB & 1387 & 405 (29.2) & Ref & Ref \\
\hline \multicolumn{5}{|l|}{ TB treatment category } \\
\hline Previously treated & 406 & $167(41.1)$ & 1.6 (1.3 to 2.0$)$ & $1.7(1.4 \text { to } 2.1)^{*}$ \\
\hline New & 4118 & $1249(30.3)$ & Ref & Ref \\
\hline \multicolumn{5}{|l|}{ Regionł } \\
\hline Eastern & 1321 & $416(31.5)$ & 1.2 (1.0 to 1.5$)$ & $1.2(1.1 \text { to } 1.5)^{*}$ \\
\hline Central & 2017 & 677 (33.6) & 1.3 (1.2 to 1.6$)$ & $1.2(1.0$ to 1.4$)$ \\
\hline Western & 1186 & $323(27.2)$ & Ref & Ref \\
\hline
\end{tabular}

*Statistically significant.

†Source of information on TB diagnosis.

‡Based on socioeconomic development level: high level in eastern region, medium level in central region and low level in western region.

PTB, pulmonary TB; TB, tuberculosis.

While TB dispensaries and designated hospitals in eastern China experience a high workload, elsewhere, particularly in western China, distances to health facilities may limit access to TB-designated hospitals for diagnosis and treatment. We propose conducting health care-seeking behaviour assessments in the western provinces to better understand possible barriers to reaching TB dispensaries and designated hospitals. Specific strategies to address TB under-reporting and timely treatment registration will likely be needed for the different socioeconomic regions in China.

Our project has a few limitations. For this initial project to evaluate TB under-reporting, we used purposive sampling to select nine countries from the 2851 counties in China. We attempted to limit the potential bias from this sampling approach by including counties from provinces that are located in three distinct regions of socioeconomic development (ie, eastern, central and western regions). However, our findings are unlikely to be representative of other locations in China. Because of the sampling approach, we must also be cautious not to overinterpret the results from our logistic regression models. Additionally, our linkage methods relied on several approaches to improve the sensitivity of finding matches between the medical records and TBIMS. Inaccurate recording or reporting by health facilities could have negatively impacted this process. Similarly, we were unable to determine the number of patients with TB who may have initiated, but were not formally registered for, treatment by the treatment facility.

Finally, national guidelines require all healthcare facilities to report both presumptive and diagnosed TB cases to the national infectious disease surveillance system. However, in this study, we focused on healthcare facilities with TB diagnostic capability and that reported at least one TB case during the study period. We did not evaluate under-reporting and timely treatment registration for facilities not meeting these criteria. Additional work is needed to determine the status of patients seeking care at facilities without TB diagnostic capabilities. Ensuring that 
these patients are diagnosed and provided with appropriate treatment is essential for reducing the overall $\mathrm{TB}$ burden in China.

\section{CONCLUSION}

We identified gaps in reporting and treatment registration in the nine counties participating in this project. The TB burden in these locations may be underestimated by almost $20 \%$. We strongly recommend training and improved guidance for reporting in high-burden regions and high-case load hospitals as well as for paediatric patients and patients with TB pleurisy. Patient education and follow-up by diagnosing facilities could help improve timely treatment registration, particularly for migrant workers and sputum-negative patients. We recommend that similar studies to assess factors associated with under-reporting and delay in registration are conducted elsewhere in China.

\section{Author affiliations}

${ }^{1}$ National Center for Tuberculosis Control and Prevention, Chinese Center for Disease Control and Prevention, Beijing, China

${ }^{2}$ South-East Asia Regional Office, International Union Against Tuberculosis and Lung Disease (The Union), New Delhi, India

${ }^{3}$ International Union Against Tuberculosis and Lung Disease (The Union), Paris, France

${ }^{4}$ Department of Medical Research (Pyin 0o Lwin Branch), Ministry of Health and Sports, Pyin 00 Lwin, Myanmar

${ }^{5}$ Division of Global Health Protection, United States Centers for Disease Control and Prevention (CDC), Beijing, China

Acknowledgements We thank Dr Yuan Li, Dr Deanna Tollefson and Dr Carol Rao for support in conducting this project and drafting the manuscript. We thank US CDC Division of Global Health Protection and the Division of Global HIV/AIDS and TB for providing technical assistance and reviewing this manuscript. We thank Dr Xiaopeng Qi and Dr Yujie Meng for generating the demonstration map. We also thank our colleagues and healthcare workers who contributed to the project from Guangxi, Henan, Heilongjiang, Hubei, Shanghai, Sichuan, Yunnan, Zhejiang and Jiangsu for data collection and review. This research was conducted through the Structured Operational Research and Training Initiative (SORT IT), a global partnership led by the Special Program for Research and Training in Tropical Diseases at the World Health Organization (WHO/TDR). The model is based on a course developed jointly by the International Union Against Tuberculosis and Lung Disease (The Union) and Medécins sans Frontières (MSF/Doctors Without Borders). The specific SORT IT programme which resulted in this publication was jointly developed and implemented by: The Union South-East Asia Office, New Delhi, India; the Center for Operational Research, The Union, Paris, France; The Union, Mandalay, Myanmar; the Operational Research Unit (LUXOR), MSF Brussels Operational Center, Luxembourg; Institute of Medicine, University of Chester, UK; and Department of Medical Research, Ministry of Health and Sports, The Republic of The Union of Myanmar

Contributors TL, HDS and KTS were involved in conception and study design. TL was involved in data extraction. TL, HDS, KTS, XD, JJR, HZ and LW were involved in analysis and interpretation of data. $\mathrm{XD}$ and $\mathrm{LW}$ were involved in overall supervision. HDS and KTS are involved as mentors. TL is the first author. XD and LW contributed equally to the article as senior authors.

Funding This project was funded by the China-US Collaborative Program on Emerging and Re-emerging Infectious Diseases (grant number (2014-2015 and 2015-2016)-3.1). The training programme was funded by the Department for International Development (DFID), UK. Project implementation was funded by the US CDC International Emerging Infections Program cooperative agreement. The study implementation as well as the open access fee for the publication is funded by the US CDC Atlanta through Combating Antimicrobial Resistant Bacteria (CARB) funds.

Disclaimer The funders had no role in study design, data collection and analysis, decision to publish or preparation of the manuscript.
Competing interests None declared.

Patient consent for publication Not required.

Ethics approval We obtained approval for this project from the Ethics Committee of Chinese Center for Disease Control and Prevention (№ 201704) and the Ethics Advisory Group of International Union Against Tuberculosis and Lung Disease (The Union) Paris, France (No 15/17, 4 April 2017). We also obtained approval from the National Center for Tuberculosis Control and Prevention of China (NCTB). The project was reviewed and approved by the US CDC. As this study involved secondary data, waiver for informed consent was sought and approved by the ethics committees.

Provenance and peer review Not commissioned; externally peer reviewed.

Data sharing statement All the data are fully available without restriction (online supplementary S1 annex).

Open access This is an open access article distributed in accordance with the Creative Commons Attribution Non Commercial (CC BY-NC 4.0) license, which permits others to distribute, remix, adapt, build upon this work non-commercially, and license their derivative works on different terms, provided the original work is properly cited, appropriate credit is given, any changes made indicated, and the use is non-commercial. See: http://creativecommons.org/licenses/by-nc/4.0/.

\section{REFERENCES}

1. World Health Organization (WHO), Global tuberculosis report 2017. WHO/HTM/TB/2017.23: Geneva, Switzerland, 2017.

2. World Health Organization (WHO). Implementing the End TB Strategy: the essentials. Geneva, Switzerland: WHO/HTM/ TB/2015.31, 2015.

3. Uplekar M, Weil D, Lonnroth K, et al. The End TB Strategy. Lancet 2015;6736:1-3

4. Lin HH, Wang L, Zhang $\mathrm{H}$, et al. Tuberculosis control in China: use of modelling to develop targets and policies. Bull World Health Organ 2015;93:790-8.

5. Huang F, Cheng S, Du X, et al. Electronic recording and reporting system for tuberculosis in China: experience and opportunities. J Am Med Inform Assoc 2014;21:938-41.

6. Wang L, Zhang H, Ruan Y, et al. Tuberculosis prevalence in China, 1990-2010; a longitudinal analysis of national survey data. Lancet 2014;383:2057-64.

7. World Health Organization (WHO). Assessing tuberculosis underreporting through inventory studies. France: WHO, 2012.

8. Tollefson D, Ngari F, Mwakala M, et al. Under-reporting of sputum smear-positive tuberculosis cases in Kenya. Int J Tuberc Lung Dis 2016;20:1334-41.

9. Fatima R, Harris RJ, Enarson DA, et al. Estimating tuberculosis burden and case detection in Pakistan. Int J Tuberc Lung Dis 2014;18:55-60.

10. Bassili A, Grant AD, El-Mohgazy E, et al. Estimating tuberculosis case detection rate in resource-limited countries: a capture-recapture study in Egypt. Int J Tuberc Lung Dis 2010;14:727-32.

11. Huseynova S, Hashim DS, Tbena MR, et al. Estimating tuberculosis burden and reporting in resource-limited countries: a capturerecapture study in Iraq. Int J Tuberc Lung Dis 2013;17:462-7.

12. China Statistics Press. China statistical yearbook 2017. $2017 \mathrm{http}: / /$ www.stats.gov.cn/tjsj/ndsj/2017/indexch.htm (accessed 5 Mar 2018).

13. Ministry of Health. Guidelines for Implementing the National Tuberculosis Control Program in China (2008). Beijing: Press of Chinese Peking Union Medical College, 2009.

14. Ministry of Health. National tuberculosis diagnostic criteria (WS2882008). Beijing,China, 2008.

15. VAN Hest NA, Story A, Grant AD, et al. Record-linkage and capturerecapture analysis to estimate the incidence and completeness of reporting of tuberculosis in England 1999-2002. Epidemiol Infect 2008;136:1606-16.

16. Bassili A, Al-Hammadi A, Al-Absi A, et al. Estimating the tuberculosis burden in resource-limited countries: a capture-recapture study in Yemen. Int J Tuberc Lung Dis 2013:17:462-7.

17. van Hest NA, Smit F, Baars HW, et al. Completeness of notification of tuberculosis in The Netherlands: how reliable is record-linkage and capture-recapture analysis? Epidemiol Infect 2007;135:1021-9.

18. Satyanarayana S, Nair SA, Chadha SS, et al. From where are tuberculosis patients accessing treatment in India? Results from a cross-sectional community based survey of 30 districts. PLoS One 2011;6:e24160.

19. World Health Organization (WHO). Global tuberculosis report 2016. Geneva, Switzerland: WHO/HTM/TB/2016.13, 2016. 
20. Chen W, Zhang $\mathrm{Q}$, Renzaho AMN, et al. Social health insurance coverage and financial protection among rural-to-urban interna migrants in China: evidence from a nationally representative crosssectional study. BMJ Glob Health 2017;2:e000477.

21. Dodd PJ, Gardiner E, Coghlan R, et al. Burden of childhood tuberculosis in 22 high-burden countries: a mathematical modelling study. Lancet Glob Health 2014;2:e453-9.

22. Marais BJ, Gie RP, Schaaf HS, et al. The spectrum of disease in children treated for tuberculosis in a highly endemic area. Int $J$ Tuberc Lung Dis 2006;10:732-8.

23. Wu X, Xu B, Jiao A, et al. The clinical epidemiological characteristics of pediatric tuberculosis in Beijing Children's Hospital from 2002 to 2010. Chin J Evid Based Pediatr 2012;7:19-24.

24. National Health and Family Planning Commission of China. Management standards for infectious diseases information reporting. 2015 http://www.nhfpc.gov.cn/jkj/s3577/201511/f5d2ab9a5e104481 939981c92cb18a54.shtml (accessed 3 May 2018).

25. World Health Organization (WHO). Guideline for drug suspectible tuberculosis and patient care. Geneve,Switzerland: WHO/HTM/ TB/2017.05, 2017.

26. Wandwalo ER, Mørkve O. Delay in tuberculosis case-finding and treatment in Mwanza, Tanzania. Int J Tuberc Lung Dis 2000;4:133-8.

27. Adenager GS, Alemseged F, Asefa $\mathrm{H}$, et al. Factors Associated with Treatment Delay among Pulmonary Tuberculosis Patients in Public and Private Health Facilities in Addis Ababa, Ethiopia. Tuberc Res Treat 2017;2017:1-9.
28. Sreeramareddy CT, Qin ZZ, Satyanarayana S, et al. Delays in diagnosis and treatment of pulmonary tuberculosis in India: a systematic review. Int J Tuberc Lung Dis 2014;18:255-66.

29. Zhou C, Chu J, Geng H, et al. Pulmonary tuberculosis among migrants in Shandong, China: factors associated with treatment delay. BMJ Open 2014;4:e005805.

30. Zhao Y, Zhang T, Liu W, et al. Investigation on health seeking delay and relevant factors among pulmonary tuberculosis patients in Shaanxi. China Trop Med 2017;17:289-92.

31. Zhao Y, Xu S, Wang L, et al. National survey of drug-resistant tuberculosis in China. N Engl J Med 2012;366:2161-70.

32. Wei X, Chen J, Chen $\mathrm{P}$, et al. Barriers to TB care for rural-to-urban migrant TB patients in Shanghai: a qualitative study. Trop Med Int Health 2009;14:754-60.

33. Peng $\mathrm{Y}$, Chang $\mathrm{W}$, Zhou $\mathrm{H}$, et al. Factors associated with healthseeking behavior among migrant workers in Beijing, China. BMC Health Serv Res 2010;10:69.

34. Mou J, Griffiths SM, Fong H, et al. Health of China's rural-urban migrants and their families: a review of literature from 2000 to 2012. Br Med Bull 2013;106:19-43.

35. Yewu Z, Qing G, Chunxi Z, et al. Application of probabilistic record linkage method in communicable disease reporting information matching. Dis Surveill 2015;30:792-5.

36. DuVall SL, Kerber RA, Thomas A. Extending the Fellegi-Sunter probabilistic record linkage method for approximate field comparators. J Biomed Inform 2010;43:24-30. 